\section{The views of dentists on their participation in a primary care-based research trial}

\author{
L. Hopper, ${ }^{1}$ A. Aspinall, ${ }^{2}$ I. C. Mackie ${ }^{3}$ and F. A. Blinkhorn ${ }^{4}$
}

IN BRIEF

- Future research in primary care should be focused on topics primary care practitioners feel of use to their clinical practice.

- Funding and protected time is necessary for primary care dental practitioners to complete research.

- There is a need for collaborative working between academic institutions and primary care dentists.

\begin{abstract}
Aims To determine the attitudes and opinions of dentists undertaking research in primary care dental practices, about the value of research in primary care. Method The data were collected during a face-to-face interview utilising a schedule of open and closed questions, which were used to guide the interview. Results Twenty-seven of the 40 primary care dentists (67.5\%) who had participated in a five-year primary care clinical trial completed the interview. All the respondents believed that primary care research was important and should be of relevance to their practice, but only 12 (44\%) thought it was important they carried out this research. The majority (96\%) of respondents felt it did not pay financially to do research as a primary care practitioner and $12(44 \%)$ thought research in primary care was too time consuming, but 25 (92\%) were prepared to get involved in a primary care research group. Five (56\%) salaried service (SS) practitioners and 14 (78\%) general dental services (GDS) dentists felt they did not have enough experience, skills or knowledge to conduct their own research. Significantly more salaried dentists compared with GDS dentists felt adequately supported to do primary care research. Conclusion Future research in primary care should be focused on topics primary care practitioners feel of use to their clinical practice. A method of funding practitioners and providing time for them to complete research away from their clinical duties is necessary. There is a need for collaborative working with an academic institution, which can provide training and academic support for practitioners.
\end{abstract}

\section{INTRODUCTION}

Primary care dental practitioners provide approximately $90 \%$ of the dental care in the United Kingdom. ${ }^{1}$ Despite the high level of clinical activity, a recent review by Clarkson in 2005 reported that only $2 \%$ of the current published research relates to primary dental care. ${ }^{1}$ In addition, the credibility of this small amount of research is often questionable in terms of scientific methodology. For example, Crawford ${ }^{2}$ searched the Cochrane Oral Health Group Controlled Trials Register for trials done

1*2Senior Dental Officers, Lance Burn Health Centre, Salford Primary Care Trust, Churchill Way, Salford, M6 50X: ${ }^{3}$ Professorial Teaching Fellow, School of Dentistry, University of Manchester, Higher Cambridge Street, Manchester, M16 6FH; ${ }^{4}$ Oral Health Promotion Coordinator, Macquarie Hospital, Northern Sydney Central Coast Area Health Service, Australia

${ }^{*}$ Correspondence to: Dr Louise Hopper

Email: louise.hopper@salford-pct.nhs.uk

\section{Online article number E4}

Refereed Paper - accepted 29 February 2008

DOI: 10.1038/sj.bdj.2008.584

${ }^{\circ}$ British Dental Journal 2008; 205: E4 exclusively in primary care and identified 174 articles. However 134 were excluded and only 43 randomised controlled trials (RCTs) met the inclusion criteria. So it is clear more effort is required to improve the design of primary care research projects.

More well-designed practice-based research should be encouraged, as this is ultimately where the majority of dental care is provided and therefore it is the most relevant place to test materials, instruments and techniques. This point was emphasised in the 1997 Mant report, ${ }^{3}$ which recognised that research undertaken in secondary care does not translate well into primary care. There is a definite need to involve primary care health professionals in research. It has also been suggested by Wilson ${ }^{4}$ that evaluation of clinical outcomes for general practitioners (GPs) should be conducted by GPs, as management decisions taken by primary care practitioners will be different to those in secondary care, reflecting different experience and priorities.

Whilst these suggestions are appealing on a practical level, it is important to remember that new legislation (EU Directive) ${ }^{5}$ means that research on humans is governed by a complex legislative framework. For research to be both legally and scientifically appropriate, it will require a partnership between academic and primary care practitioners. Crawford ${ }^{2}$ recently alluded to the importance of how best to recruit dentists to participate in practice research and suggested that more information is required on how dentists involved in offering clinical care perceive their research role.

Although research is seen as an important activity for health professionals, the dental literature reveals some contradictions. Kay et al. ${ }^{6}$ found GDPs were keen to be involved with practice research networks and found the experience very useful, whilst Blinkhorn et al. ${ }^{7}$ found research groups less successful. 
Of 872 dentists contacted to recruit to a primary care research network, only 98 attended and out of these, only one request for help with a research project was received.

Although there are many articles evaluating views of GDPs on research, ${ }^{6-8}$ it is interesting to note that there is no published literature on the views of primary care dentists who have already taken part in a clinical trial. By targeting this group of practitioners and investigating their views on participating in an actual trial, a clearer view on the real barriers could be identified, rather than assessing perceptions of barriers from people who have not been actively involved in primary care research. By approaching the problem in this way and gaining their views and opinions, concrete suggestions on how to improve the training and recruitment of GDPs to undertake practice-based research can be formulated.

\section{METHOD}

Forty primary care dentists who had participated in a five-year clinical trial testing pulp treatment medicaments in primary teeth were contacted.

The dentists were all located in the North West region of England and had been involved in a five-year clinical trial funded by the National Primary Dental Care Research and Development Programme (RD0/20/22). Recruitment for this original study was by open invitation to dentists in the Greater Manchester area of North West England. There were many different venues in a range of social settings and a variety of clinicians involved in the study.

The dentists were contacted by a research assistant who undertook a faceto-face structured interview. All data were anonymised so that there would be no constraints on participation. The interview was structured around three themes, namely:

1. Training, funding and support

2. Importance and relevance of research

3. Time factors and pressures of research in primary care.

Two groups of dentists were identified: salaried services (SS) and general dental services (GDS). The project had ethical approval from the Central Manchester Local Research Ethics Committee.

\section{RESULTS}

Forty primary care dentists were recruited and they worked in general dental practice or personal dental service and community dental clinics. Twenty-seven (67.5\%) were available for interview at the end of the fiveyear trial: out of 12 SS dentists in the original trial, nine (75\%) completed the interview; out of 28 GDS dentists, $18(64 \%)$ participated.

The majority of SS staff were female (89\%), whilst the majority of GDS staff were male $(72 \%)$.

\section{Training, funding and support}

There was a feeling of lack of experience in research amongst both SS and GDS dentists, with no significant difference between the two groups. They did not feel sufficiently confident to conduct their own projects: five (56\%) SS practitioners and $14(78 \%)$ GDS practitioners felt they did not have enough experience, with two GDS dentists commenting 'we do not have the necessary skills or knowledge'.

Six (67\%) of the SS practitioners felt competent to organise their own primary care research compared to six (33\%) of the general practitioners, with four GDS and two SS dentists expressing a feeling of lack of understanding on how to proceed for ethics committee approval and a lack of knowledge on how to design the trials. Typical comments were 'ethics committee approval is very complicated', and 'ideas practitioners have do not get approval because of flaws in methodology'. There was no significant difference between the two groups.

Significantly more SS dentists (8 [89\%]) felt they were adequately supported to carry out primary care research compared to two (11\%) GDS dentists ( $\mathrm{p}<0.001)$.

Support for the idea of a primary dental care research group was almost unanimous, with 25 (93\%) practitioners willing to become involved. The interest in such a group was also expressed during the interview, with a SS practitioner suggesting 'the need for a forum for GDPs to get together and organise research'. Four dentists (two SS and two GDS) expressed a need for personnel support and an increase in their knowledge on how to conduct research. Comments were made on their need for 'time, personnel and support', and 'we do not have skills or knowledge' to conduct research, whilst one SS dentist also commented that commitments to service provision affects the availability of time to undertake research'.

All of the practitioners bar one (96\%) felt that it did not pay financially to undertake research as a primary care dentist. Comments were made on the lack of financial support and manpower and the hope that with the new GDS contract it 'may make it possible to do funded research - if the government recognise it!'

\section{Importance and relevance of research}

Less then half (12 [44\%]) of the primary care practitioners thought that it was important that they carried out research, with eight (30\%) practitioners disagreeing that they should carry out the research. One of the SS dentists commented that the 'best research is carried out by university staff, it's their job to do it!' There was no significant difference between the views of the SS and GDS dentists.

All of the practitioners interviewed thought it was important that research should be relevant to primary care, with two GDS practitioners commenting that 'research in primary care must grow - it's where 95\% of the treatment is done and research needs to be relevant to it' and another GDS dentist commenting that they 'would like to see more research done in primary care'. They were only interested in getting involved in studies if they were 'relevant and interesting' to them. There was no significant difference between the views of the two groups.

\section{Time factors and pressures of research in primary care}

Twelve (44\%) of the dentists interviewed thought that research in primary care was too time consuming, eight (30\%) disagreed with this and the remaining dentists held a neutral stance, with no significant difference between the SS and GDS dentists. 
Twenty-two (82\%) of the dentists felt that the pressures of practice made it hard to carry out research as well as their clinical commitments. Four dentists (two GDS and two SS) commented on the need for time for research to be separate from their practice commitments, with comments such as 'SS commitments to service provision affect the time available'.

Regarding the length of research trials and the ability for primary care practitioners to commit to lengthy trials, six dentists (22\%) thought primary care practitioners changed jobs too frequently to be involved in longitudinal studies. Eight dentists (30\%) did not have an opinion and 13 dentists (48\%) disagreed and felt dentists did not move jobs too frequently to become involved with lengthy trials. No significant difference between the SS and GDS dentists was found. However, almost one third of the sample (32.5\%) was not contactable at the end of the trial because they had changed jobs!

When asked about future involvement in research and duration of the trial, all the dentists said they would become involved in a study of shorter duration. Six (67\%) SS dentists and 11 (61\%) GDS dentists said they would be involved in a trial of similar duration to the just completed five-year trial. However only eight $(30 \%)$ of the dentists questioned would be willing to become involved in a trial of longer duration, three (33\%) from the SS and four (22\%) from the GDS. There was no significant difference between the two groups of practitioners.

\section{DISCUSSION}

The problems of recruiting dentists to primary care research has been well documented in the past few years, with a lack of interest, lack of support, lack of sufficient skills, patient factors, lack of time and lack of remuneration being cited as possible causes. ${ }^{6,9-11}$

However, some groups of researchminded GDPs have flourished, such as the Clinical Research Associates in the USA, the PREP panel, a UK-wide group of 30 GDPs, and BRIDGE, a West Midlands-based group of practitioners. These groups have been running for a number of years and have maintained an output of research carried out in primary care. For example, the PREP panel have completed over 50 projects (www.preppanel.com).

One of the main points highlighted by this study is the need for training of some primary care practitioners in research, and support for all by academic institutions. Even amongst a group of motivated, interested practitioners who had already participated in a five-year trial, there was a general lack of knowledge on how to conduct their own projects, with specific comments on research methodology and a lack of knowledge on how to proceed for ethics committee approval. This need for training has been identified in other papers ${ }^{1,6,8,10-12}$ where it was concluded that for practice-based research to develop, practitioners must develop research skills, as well as being research aware or having the close support of an academic-based research team. ${ }^{8,13}$

Significantly more SS dentists felt they had support to carry out research when compared to GDS dentists. This could be due to the SS dentist having more resources and support available to them through their primary care trusts. For example:

- Funding for postgraduate study

- Access to library facilities

- Active research being undertaken by members of the SS team

- Links between the SS and academic institutions.

Usually senior dental officers (SDOs) in the SS have a postgraduate qualification with a research component, yet not all of the SS dentists in this study who felt more competent had postgraduate qualifications.

Another reason could be attributed to the fact that SS dental posts are salaried, meaning dentists might find it easier to take time out to do the necessary paper work or research the necessary approval networks. More research would need to be done to prove this point. However, one SS dentist did comment that 'commitments to service provision affects the time available' for research. This comment is becoming realistic of the situation in the future for the salaried services, as PCTs now have targets to meet and time and research resources may become fewer in the future.
Although this feeling of lack of knowledge and support has been identified in other papers as a barrier to research, ${ }^{6} \mathrm{a}$ difference between the SS and GDS has not previously been shown.

This study showed that primary care dentists think that research is important to their practice of dentistry. The dentists who participated in this trial were prepared to become involved in further projects if they were 'relevant and interesting' and had a perceived relevance to their clinical practice. These findings support the suggested barriers shown by other studies, namely that primary care practitioners are research aware and recognise the importance of primary care research and that their interest in research is influenced by the relevance of the research to their practice. ${ }^{1,9,14}$ In primary care medical practice, Ward found that the level of personal interest influenced general medical practitioners' participation in trials. ${ }^{15}$

Ownership of the trial was discussed in a paper by Jones et al., who suggested that GDPs should be more closely involved in all aspects of the trial design so they feel more involved from the outset. ${ }^{16}$

This study showed a range of views on whose duty it is to carry out research. One of the dentists commented that the 'best research is carried out by university staff, it's their job to do it!' Thus, a possible reason for the problem with recruiting primary care dentists to carry out trials is that they do not feel that it is their job. This was highlighted in the Department of Health's Reflection and Moving Forward conference. ${ }^{17}$

This study shows that the length of the trial is an important factor to consider in recruiting dentists into primary care research. It is also relevant to consider the fact that out of 40 dentists involved at the beginning of the five year trial, only 27 were still working at the same place at the end and $32.5 \%$ (13) of the original 40 had moved jobs!

Palmer $^{8}$ found in a survey of Merseyside GDPs that they would be interested in participating in collaborative research if their income and time could be protected, and it was incorporated into a normal working day. Clarkson ${ }^{9}$ and Burke ${ }^{10}$ also stated lack of time in clinical practice as a barrier to practice- 
based research. Our study agrees with these conclusions. The need for 'set aside time' and personnel support was thought to be important by dentists involved in this study, but it also found that primary care dentists thought that the time commitment of a study was less important if they thought that the trial would be 'relevant and interesting.' Some primary care groups, such as PREP panel, aim for all projects to have funding to reimburse the primary care dentists' time.

It has been suggested that by minimising time commitments to trials by the use of research assistants, more primary care practitioners would become involved. ${ }^{14}$ When asked about the pressures of practice making research hard, the majority (81.5\%) agreed, showing that it is important for help to be offered by employing research assistants and formally allocating specific research sessions.

The study confirms that inadequate funding is a barrier to research being undertaken in primary dental care. ${ }^{1,8,9}$ It is important to highlight that this research was carried out when GDS dentists were still paid by fee per item, before the implementation of the new contract. A number of those dentists who worked in non-salaried posts commented that the old GDS contract did not make research financially possible and hoped that the new contract would make it easier to participate in research. It would be worthwhile repeating this research now that the new contract has been implemented to see what impact, if any, this has had on the views of SS and GDS practitioners. Solutions to this problem are to provide adequate funding. As part of the GDS contract, the PCTs could incorporate research into interested practitioners' contracts. Another option is to provide financial incentives to enter into trials. It has been shown that financial incentives do work for general medical practitioners but this creates its own problems, by raising the question as to the representability of the data to the typical population. ${ }^{14}$

The use of primary care research groups has proven to be a popular means of integrating research into primary care and providing the necessary funding, academic support and training for the practitioners. Examples of this have been the primary dental care research and development funding initiative set up in 1996. A recent paper by Kay et $a l .{ }^{6}$ describes practitioners' experience of being part of the North West primary care research group. The results showed that all the involved practitioners wished to continue with the group and the participating dentists derived immense personal and professional fulfilment from being part of a group with a common aim. However Kay et al. ${ }^{6}$ did not report what positive research outcomes in the form of research reports had been published by the group.

Our study also confirmed that setting up a primary care research group would be popular with the individuals who had been involved in the five-year clinical trial. The dentists wanted support, and primary care network groups would be a good way of providing new ways of collaborative working, of benefit to both primary care practitioners and academic institutions in order that high quality primary care research can be undertaken.

There are a number of limitations to this study: there were only 27 dentists who participated in the structured interviews, but their views are important as they had actually participated in a primary care research project. It could be argued that as they had been involved in a five-year trial, they were more research aware and might not be representative of primary care practitioners as a whole. Nevertheless, their views and problems were similar to other studies.

\section{CONCLUSIONS}

This study has shown that to encourage more primary care dentists to actively participate in research, adequate time, funding and support needs to be set aside.

This study also shows that dentists are less keen to participate in trials of longer than five years duration.

Primary care dentists from both the SS and GDS do not feel they have adequate knowledge or skills to carry out their own research in primary care. Support and training is needed from academic institutions. It is the view of this study that this is best provided by primary care research being a collaboration between primary care clinicians and academic research teams, possibly through the use of research assistants.

Statistically significantly more SS dentists compared with GDS dentists feel they receive adequate support to do primary care research.

We would like to thank those primary care dentists who were involved in the pilot study and the trial, Mrs Christine Mackie who undertook the interviews and Professor Helen Worthington for her advice regarding data analysis. This study was funded by the National Primary Dental Care R\&D Programme, reference number RDO/90/22.

1. Clarkson J. Experience of clinical trials in general dental practice. Adv Dent Res 2005; 18: 39-41.

2. Crawford F. Clinical trials in dental primary care: what research methods have been used to produce reliable evidence? Br Dent J 2005; 199: 155-160.

3. Mant D. Research and development in primary care. National working group report. London: Department of Health, 1997.

4. Wilson S, Delaney B C, Roalfe A et al. Randomised controlled trials in primary care: case study. BMJ 2000; 321: 24-27

5. Directive $2001 / 20 /$ EC of the European Parliament and of the Council of 4 April 2001, on the approximation of the laws, regulations, and administrative provisions of the member states relating to the implementation of good clinical practice in the conduct of clinical trials on medicinal products for human use. Official Journal European Communities 2001; May 1: L121/44.

6. Kay E, Ward N, Locker D. A general dental practice research network - philosophy, activities and participant views. Br Dent J 2003; 194: 545-549.

7. Blinkhorn A S, Clarkson J E, Craven R, Holloway $\mathrm{PJ}$, Worthington H V. An evaluation of a training programme in primary care research for general dental practitioners. Br Dent J 2000; 188: 333-336.

8. Palmer N, Grievson B. An investigation into Merseyside general dental practitioners' interest in primary care research, their views on research and their training needs. Primary Dent Care 2005; 12: 145-149.

9. Clarkson J E. Getting research into clinical practice - barriers and solutions. Caries Res 2004 38: 321-324.

10. Burke $F$ M, O'Mullane D. Oral research in primary care. Adv Dent Res 2005; 18: 37-38.

11. Hopkins $L M$, Eaton $K A$. Research in general dental practice - what is involved? Part 1: general considerations. Primary Dent Care 1996; 3: 71-74.

12. Hichens L P, Sandy J R, Rowland H N et al. Practical aspects to undertaking research in the primary care setting: experience from two studies. J Orthod 2005; 32: 262-268.

13. Innes N P T, Evans D, Clarkson J, Foley J. Obtaining an evidence base for clinical dentistry through clinical trials. Primary Dent Care 2005; 12: 91-96.

14. Foy R, Parry J, McAvoy B. Clinical trials in primary care: targeted payments for trials might help improve recruitment and quality. BMJ 1998: 317: 1168-1169.

15. Ward J. General practitioners' experience of research. Fam Pract 1994; 11: 418-423.

16. Jones C S, Billington R W, Pearson G J. The advantages and disadvantages of running a clinical trial in general practices. Br Dent J 2004; 197: 311-313.

17. Department of Health. National programme for primary dental care research and development. Reflection and moving forward. London: Department of Health, 2004. 